\title{
A Hidden Order: Exploring the Relationship of Music and Art through Geometry
}

\author{
Sama Mara \\ Musical Forms \\ UK \\ www.samamara.com \\ sama@musicalforms.com
}

\section{INTRODUCTION}

This paper serves to give an overview of the audiovisual project $A$ Hidden Order by artist Sama Mara and composer Lee Westwood. The project explores the relationship between music and art, and is founded upon a method discovered by Mara that enables the translation of music into geometric art and vice versa. Within this method pattern relates to rhythm in a one to one relationship, and colour relates to pitch (Mara 2016). The basic principle of this method is founded upon the observation that number is the basis of harmony for both music and art, so it is through mathematics and geometry that these fields may be brought together.

The use of this theory enables the creative processes of one discipline to be directly implemented in the other. For example one could compose a piece of music considering melody, dynamics and structure of the piece and they will be directly represented in the visual counterpart. One might also start from a visual design, selecting particular colours and motifs which are then translated into music. The composition process may involve a conversation going back and forth between the two disciplines to arrive at a final piece that has been developed from the creative processes of both music and visual art.

The resultant pieces are at once a musical and a visual work as the co-creator of $A$ Hidden Order Lee Westwood describes it: '...it should be remembered that, whether we are looking at the image, or we are hearing the musical composition, we are, in fact, perceiving the very same artistic "object", from a different angle, or through the eyes of a different medium (sound or visual pattern)'.

The musical style traverses the realms of contemporary composition, with the striking syncopations and harmonies of folk and jazz, whilst the resultant aesthetic has strong resonances with traditional Islamic geometric art and modern fractal geometry.

\section{CREATIVE PROCESS}

The composition and design stage of the project involved a variety of approaches from pure musical composition, to an interplay between the musical and the visual considerations, to purely visual design.

On completion of the composition / design stage the resultant pieces were rehearsed and professionally recorded by a quartet of musicians comprised of cor anglais, flute, marimba \& percussion, and cello. Using a bespoke computer programme, built around the method described, these recordings were translated into their geometric counterparts in the form of a series of digital prints and short films.

\section{OUTCOMES}

The nature of the project, spanning both visual arts and music meant that the final project resulted in various forms including:

- A live audio-visual performance

- A Collection of giclée prints

- A Film suite

- An Interactive platform

- An Album

\section{REFERENCES}

Kandinsky, W. (1914) Concerning the Spiritual in Art. Dover Publications Inc. (1977), p. 25.

Mara, S. (2016) The Rhythm of a Pattern. Bridges Finland 2016. The University of Jyväskylä, 9-13 August, pp. 309-316. Tessellations Publishing, Phoenix, Arizona, USA. 

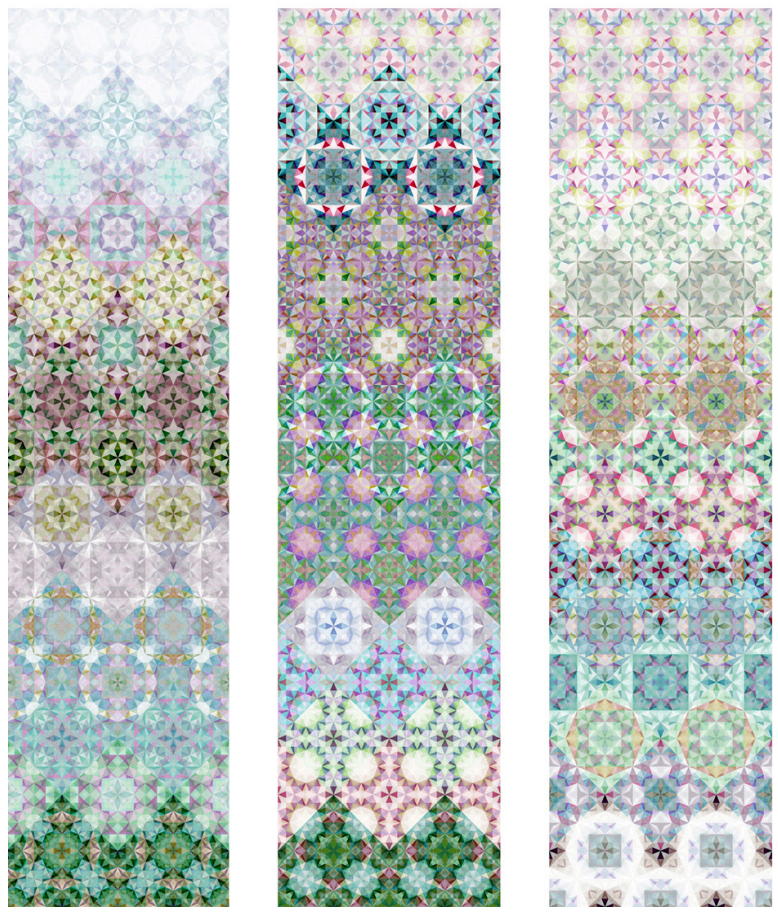

Figure 1: Octagon Square - Marimba. An example artwork from the A Hidden Order collection.

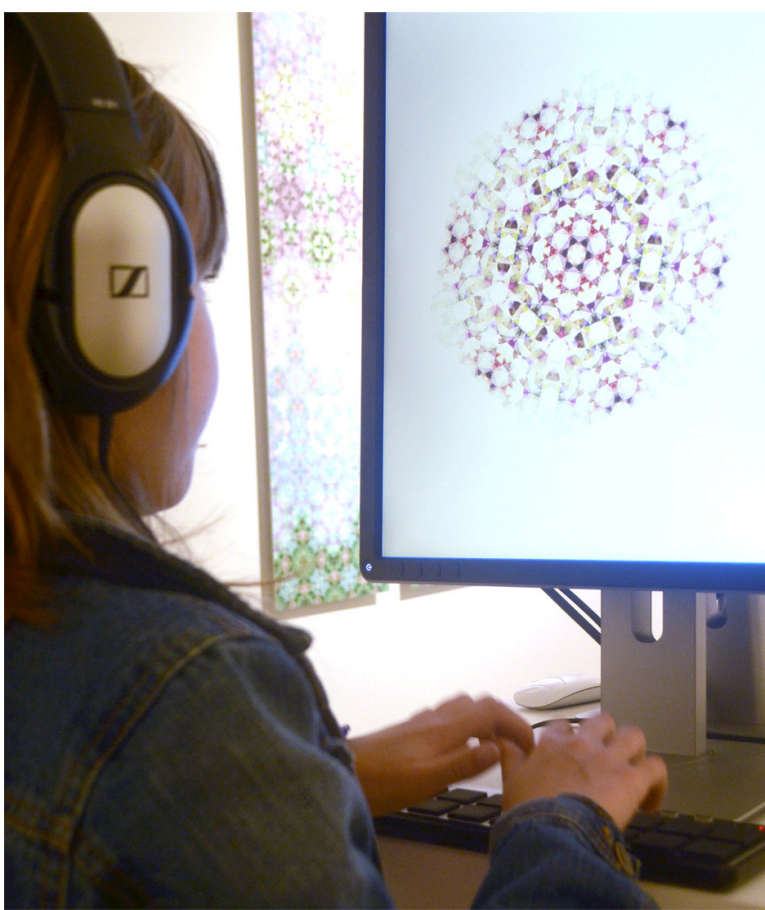

Figure 2: The interactive platform, where the user may create patterns by making music through a simple musical interface.

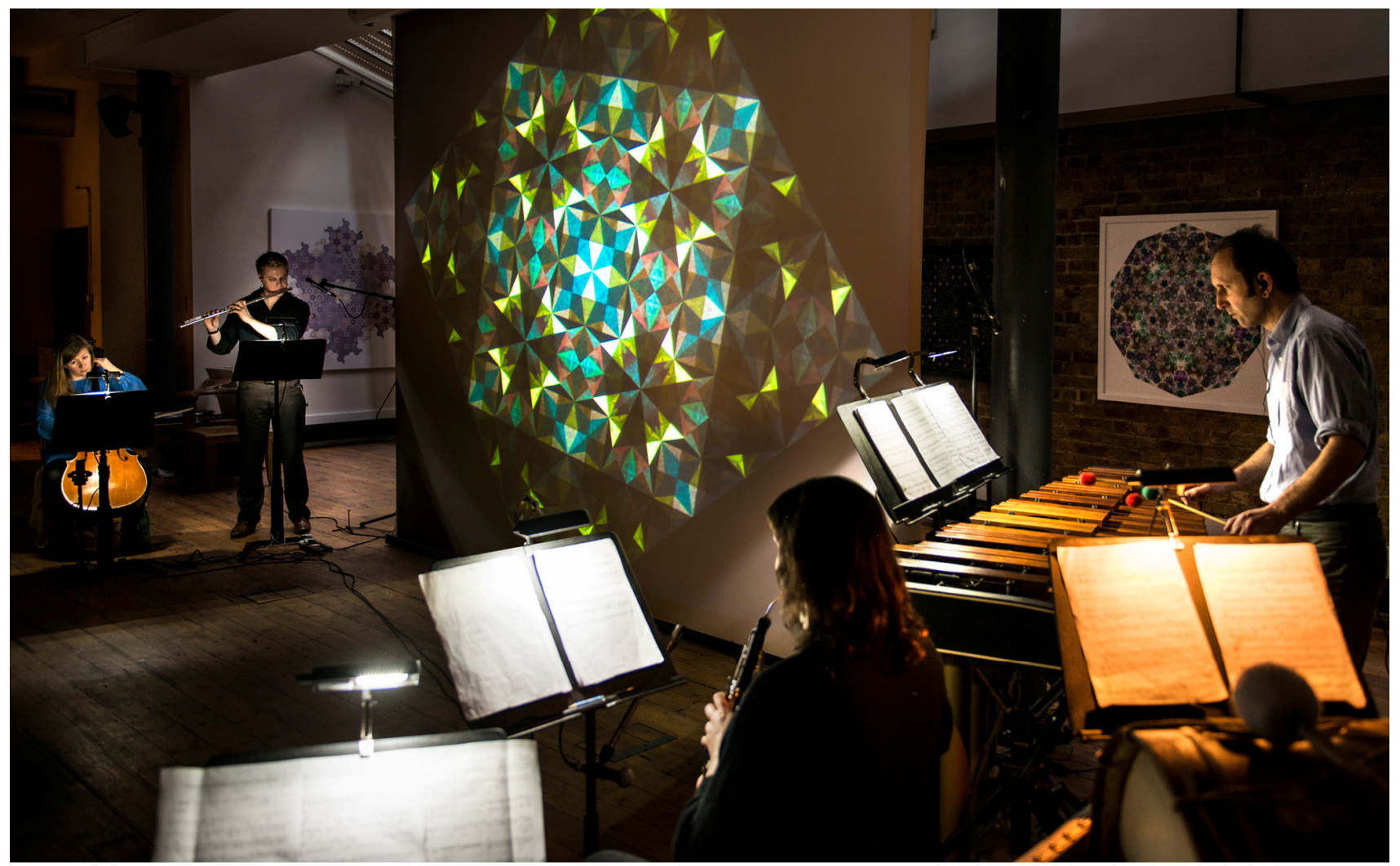

Figure 3: The live audio-visual performance of A Hidden Order. 